\title{
Normal Mode Analysis of KRas4B Reveals Partner Specific Dynamics
}

\author{
Published as part of The Journal of Physical Chemistry virtual special issue "Ruth Nussinov Festschrift". \\ Meryem Eren, Nurcan Tuncbag, Hyunbum Jang, Ruth Nussinov, Attila Gursoy, and Ozlem Keskin*
}

Cite This: J. Phys. Chem. B 2021, 125, 5210-5221

Read Online

\section{ACCESS | Llll Metrics \& More | 喟 Article Recommendations | S1 Supporting Information}

ABSTRACT: Ras GTPase interacts with its regulators and downstream effectors for its critical function in cellular signaling. Targeting the disrupted mechanisms in Ras-related human cancers requires understanding the distinct dynamics of these proteinprotein interactions. We performed normal mode analysis (NMA) of KRas4B in wild-type or mutant monomeric and neurofibromin-1 (NF1), Son of Sevenless 1 (SOS1) or Raf-1 bound dimeric conformational states to reveal partner-specific dynamics of the protein. Gaussian network model (GNM) analysis showed that the known KRas4B lobes further partition into subdomains upon binding to its partners. Furthermore, KRas4B interactions with

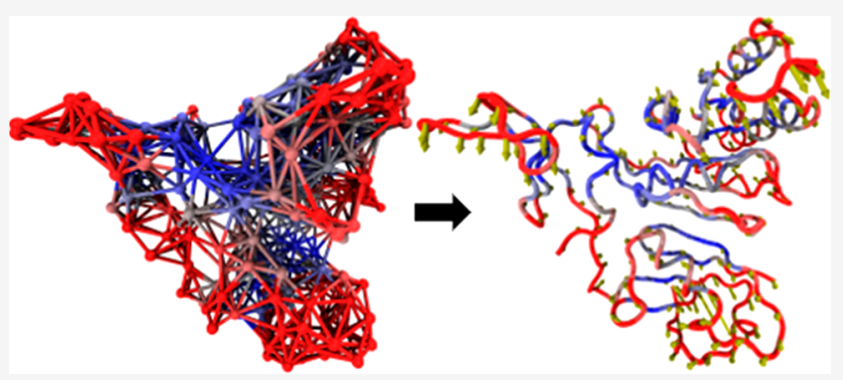
different partners suppress the flexibility in not only their binding sites but also distant residues in the allosteric lobe in a partnerspecific way. The conformational changes can be driven by intrinsic residue fluctuations of the open state KRas4B-GDP, as we illustrated with anisotropic network model (ANM) analysis. The allosteric paths connecting the nucleotide binding residues to the allosteric site at $\alpha 3$-L7 portray differences in the inactive and active states. These findings help in understanding the partner-specific KRas4B dynamics, which could be utilized for therapeutic targeting.

\section{INTRODUCTION}

Ras proteins are a family of small guanosine triphosphatases (GTPases) and have a conserved catalytic domain (G-domain) for activity. They are active when GTP is bound and inactive when guanosine diphosphate (GDP) is bound. ${ }^{1}$ The GTPase activating proteins (GAPs) speed the intrinsically slow GTP hydrolysis reaction and take Ras to its inactive state. ${ }^{2}$ Guanosine exchange factors (GEFs) activate Ras proteins by catalyzing the removal of GDP from the protein's nucleotidebinding site, resulting in GTP binding. ${ }^{2}$ GTP-bound Ras activates various signaling pathways crucial to cell proliferation and survival by interacting with effectors like Raf, phosphoinositide 3-kinase (PI3K), and Ral guanine nucleotide dissociation stimulator (RalGDS) at their Ras binding domains (RBDs). ${ }^{3}$ Mutant Ras proteins with constitutively active state promote tumorigenesis by hyperactivation of the downstream signal pathways. ${ }^{3,4}$ Human RAS isoforms are HRAS, KRAS, and NRAS. KRAS oncogenes make up about $82 \%$ of the Ras nonsynonymous mutations seen in human cancer according to data from COSMIC. ${ }^{5}$ The clinical importance of KRas protein makes the dominant splice variant KRas4B the focus of our study.

Figure 1A shows the KRas4B structure in active state together with its secondary structure elements. The G-domain (residues 1-166) is divided into two lobes based on function. The effector lobe (residues $1-86$ ) is the $\mathrm{N}$-terminal region of the catalytic domain: P-loop (residues 10-17) binds GTP/
GDP with a $\mathrm{Mg}^{2+}$; Switch-I (residues 30-38) and Switch-II (residues 60-76) regions make up the main protein binding sites. ${ }^{1,6}$ The allosteric lobe (residues 87-166) contains $\alpha$-helical dimerization interface and nucleotide base binding motifs and relays information through the protein (about the interactions with the nucleotide, proteins, and membrane). ${ }^{6,7}$ The hypervariable region (HVR, residues 167-188) is posttranslationally modified and binds to the plasma membrane. ${ }^{6,8}$

KRas4B can form heterodimers with its regulators such as GEFs and GAPs or with its effectors such as Raf and PI3K. Figure 1B highlights KRas4B in complex with Son of Sevenless 1 (SOS1, Ras GEF), neurofibromin 1 (NF1, Ras GAP), and Raf-1 specifically. These partner proteins bind through different but partially overlapping regions on KRas4B. Switch-I, $\beta 2-\mathrm{L} 3-\beta 3$ are on the KRas4B-Raf-1 interaction interface. ${ }^{9}$ Both Switch regions are on the KRas4B-SOS1 and KRas4B-NF1 interaction interfaces. ${ }^{10,11}$ SOS1 inserts an $\alpha$ helical wedge structure to the nucleotide-binding site of GDPbound Ras, moves Switch-I away from GDP, and modifies Switch-II conformation to facilitate the nucleotide exit. ${ }^{10} \mathrm{NF} 1$

Received: January 31, 2021

Revised: April 26, 2021

Published: May 12, 2021 


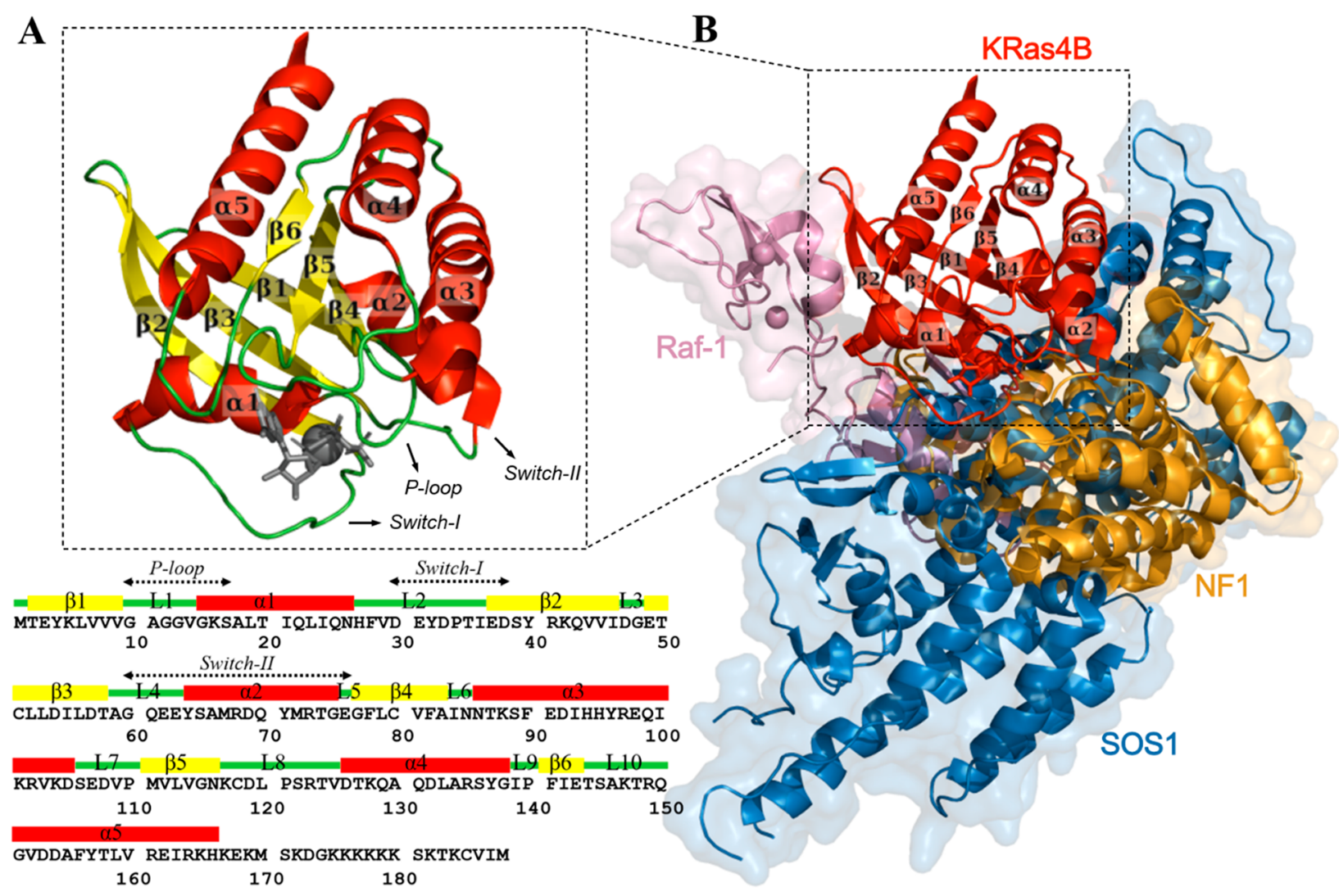

Figure 1. KRas4B structure, sequence, and protein interactions. (A) Detailed structure of active state KRas4B (6GOD_A). The $\alpha$-helices, $\beta$ strands, and loops are colored red, yellow, and green, respectively. KRas4B sequence (UniProt code P01116) and the corresponding secondary structures are shown in the lower panel. (B) Representative GEF, GAP, and effector interactions of KRas4B (red) with SOS1 (blue, 6EPL_RS), NF1 (orange, 6OB2_AB), and Raf-1 (pink, 6XI7_AB). KRas4B-SOS1 binding interface residue indices are 17, 21, 30-32, 34, 40, 54-57, 59-61, $63-71,73,102$, and 105 according to HotRegion. NF1 binding interface residue indices are 21, 25, 31-34, 36-41, 61-64, 66, 67, and 88. Raf-1 binding interface residue indices are $21,23-26,29,31,33,36-48,56,149,153$, and 157. Structure images were prepared using the PyMOL Molecular Graphics System, version 1.2r3pre, Schrödinger, LLC.

does the opposite; it interacts with Switch regions to catalyze the GTP hydrolysis reaction. ${ }^{11}$ Although GTP-bound active KRas4B can interact with GAPs and effectors; Switch-I residue positions and orientations selectively recruit them. ${ }^{12,13}$

The unique structural and biophysical properties of different KRas4B conformations affect its dynamics. There are various studies on the GTP-bound and GDP-bound states of Ras proteins that analyze the nucleotide-switching and hydrolysis reactions. ${ }^{7}$ A shift in $\alpha 3$-L7 toward the allosteric lobe enables Switch-II ordering for GTP hydrolysis. ${ }^{13}$ The reaction occurs basically through proton shuffling from a catalytic water molecule to residues Q61 and Y32 via the nucleotide's $\gamma$ phosphate and the bridging water molecule. This culminates in the remaining hydroxide ion's attack on the $\gamma$-phosphate causing formation of GDP and an inorganic phosphate. This allosteric modulation of Switch-II enables GTP hydrolysis. ${ }^{13}$ GDP-bound inactive state is associated with higher Switchregion flexibilities, while GTP binding introduces new hydrogen bonds and increases stiffness of the structure. ${ }^{14-16}$ T35 and G60 make hydrogen bonds with GTP's $\gamma$-phosphate oxygen atoms, Y32 resides over the nucleotide with a "bridging" water molecule, and also S17 and T35 side chains coordinate $\mathrm{Mg}^{2+}$ with two water molecules and the nucleotide's $\beta$ - and $\gamma$-phosphates. ${ }^{16,17}$ Hydrogen bonding network is altered after GTP hydrolysis: T35 and G60 no longer interact with the nucleotide, Y32 side chain orientation changes, and $\mathrm{T} 35$ does not coordinate $\mathrm{Mg}^{2+} \cdot{ }^{16,18}$ Therefore,
Ras is overall more flexible in the GDP-bound "open" state whereas the GTP-bound state is associated with less flexibility, especially in P-loop, $\beta 4, \beta 5$, and $\beta 6$. $^{14,15}$ Mutations can also lead to conformational and allosteric changes, affecting protein interactions. ${ }^{11,19-23}$ Therefore, comprehending the characteristics of KRas4B dynamics in different conformations can shed light on its oncogenic activity in various signal pathways. Most Ras dynamics studies are performed by atomistic molecular dynamics (MD) simulations. However, MD simulations are computationally expensive to study the overall dynamics of KRas4B with its partners. Normal mode analysis (NMA) can help in overcoming this challenge as it is computationally efficient and provides the large-scale collective motions. ${ }^{24}$ NMA was utilized in previous studies investigating the nucleotide-switch mechanism of Ras superfamily, ${ }^{15}$ the intrinsic dynamics of $\mathrm{KRas} 4 \mathrm{~B},{ }^{14,15}$ and also effects of mutations on KRas4B dynamics. ${ }^{19}$ However, there is not a comprehensive study that shows the dynamic differences when KRas4B is bound to its regulators and effectors, i.e., KRas4B partnerspecific dynamics. This could help with understanding the undruggable KRas4B dynamics toward drug design.

Gaussian network model (GNM) ${ }^{25}$ and anisotropic network model (ANM) ${ }^{26}$ are NMA-based methods and give accurate estimates on global protein motions at slow modes and local motions at fast modes. ${ }^{27}$ Residues with high fluctuations in slow modes correspond to more flexible regions, while the residues with lower fluctuations form the hinge sites critical for 
Table 1. List of Analyzed KRas4B Structures and the Associated Data

\begin{tabular}{|c|c|c|c|c|c|c|}
\hline PDB code $^{a}$ & type & resolution $(\AA)$ & binding status & main KRas chain & mutation status & nucleotide $^{b}$ \\
\hline 4OBE A & $\mathrm{X}$-ray & 1.24 & unbound & A & WT & GDP \\
\hline 6GOD A & $\mathrm{X}$-ray & 1.71 & unbound & A & WT & GNP \\
\hline 4EPR A & $\mathrm{X}$-ray & 2.00 & unbound & A & G12D, C118S & GDP \\
\hline 6GOF B & $\mathrm{X}$-ray & 1.98 & unbound & A & G12D & GNP \\
\hline 6EPL RS & $\mathrm{X}$-ray & 2.55 & SOS1 (REM-CDC25) bound & $\mathrm{R}$ & G12C, C118S, DTK126ESR & \\
\hline $6 \mathrm{OB} 2 \mathrm{AB}$ & $\mathrm{X}$-ray & 2.85 & NF1 (GRD) bound & A & WT & GNP \\
\hline 6XI7 AB & $\mathrm{X}$-ray & 1.95 & Raf-1 (RBD-CRD) bound & B & WT & GNP \\
\hline
\end{tabular}

the protein activity. ${ }^{27,28}$ Peak residues in fast modes comprise "kinetically hot residues" constrained in the structure and with roles in folding or binding. ${ }^{29,30}$ Moreover, changes in residue motions over all modes provide insight into protein's function and allosteric regulation. ${ }^{31-33}$ These network models can be further modified by incorporating residue specificity ${ }^{34}$ or used in concert with other methods such as perturbation-response scanning. ${ }^{35,36}$

In this study, we perform NMA of KRas4B complexed with SOS1, NF1, and Raf-1 to analyze its partner specific conformational dynamics. We use GNM to investigate the global and local KRas4B intrinsic residue fluctuations in the complexes. The modules of residues with correlated fluctuations may indicate functional subdomains in the structure. We employ ANM to highlight the transition from inactive "open" state of wild-type KRas4B to bound "closed" states of KRas4B with its partners, calculating theoretical residue displacements. We use a Monte Carlo path (MCPath) generation approach ${ }^{37}$ and a computationally efficient network-based method, $\mathrm{Ohm},{ }^{38}$ to find allosteric pathways that might differentiate between these conformations. As a summary, we aim to reveal how binding to different partners affects residue flexibilities, introduces new intramolecular residue communications, and forms functional subdomains in KRas4B.

\section{METHODS}

Protein Structures. We selected seven KRas4B representative structures from Protein Data Bank (PDB) with high resolution and no missing sequences based on their mutation status, bound nucleotide type, and the interacting proteins (Table 1). KRas4B proteins in all the structures were modified to start and end at the same residue, from T2 to H166.

Gaussian Network Model (GNM) with All C $\alpha$ in the Structure and the Nucleotide Atoms. Normal mode analysis was performed with ProDy. ${ }^{39}$ The parameters we used for analysis with ProDy are provided in Supporting Information (Table S1). We applied GNM analysis for the seven KRas4B structures and considered the complex forms of the proteins (see Table 1 first column for chain identifiers) while also including the nucleotides in the structures. Besides all $\mathrm{C} \alpha$ atoms, the nucleotide atoms $\mathrm{C}^{\prime}, \mathrm{C} 4^{\prime}, \mathrm{P} \alpha, \mathrm{P} \beta$, and $\mathrm{P} \gamma$ were used to define the contact maps. We set the cutoff distance to $7.5 \AA$ and obtained $N-1$ nonzero normal modes $(N=\mathrm{C} \alpha$ and nucleotide atom number). The squared fluctuations over the weighted 10 slowest modes and the individual three fastest modes were calculated for each structure to compare their global and local motions, respectively. The cross-correlations between residue fluctuations were determined from overall GNM modes. We prepared the graphics with Matplotlib ${ }^{40}$ using the normalized results. The HotRegion ${ }^{41}$ results of the complexes were obtained to determine the regions on the interaction interfaces. We also analyzed the structures in terms of the node centrality with network analysis of protein structures (NAPS) ${ }^{42}$ using default parameters in the Web server.

Experimental Distance Vectors. All structures were superimposed on the reference wild-type KRas4B-GDP structure at their common $\alpha$-helix and $\beta$-strand residues (rigid parts, 109 residues in total). The distance vector $\Delta D=$ $\left[D_{2}, D_{3}, D_{4}, \ldots, D_{166}\right]$ contains the residue displacements given by $\mathrm{C} \alpha$ displacements

$$
D_{i}=\sqrt{\left(C_{i_{x}}^{\mathrm{A}}-C_{i_{x}}^{\mathrm{B}}\right)^{2}+\left(C_{i}^{\mathrm{A}}-C_{i_{y}}^{\mathrm{B}}\right)^{2}+\left(C_{i}^{\mathrm{A}}{ }_{z}-C_{i_{z}}^{\mathrm{B}}\right)^{2}}
$$

between two conformations of the protein (B and A) ${ }^{43}$ Here, the $\mathrm{C} \alpha$ atom coordinates are represented by $C_{i}^{\mathrm{A}}$ and $C_{i}^{\mathrm{B}}$, where $\mathrm{B}$ is the reference structure coordinates and $i$ is the residue number (from 2 to 166).

Anisotropic Network Model (ANM) with Only Reference Structure $\mathrm{C} \boldsymbol{\alpha}$ Atoms. We further performed ANM analysis of the inactive state of KRas4B (GDP-bound) which corresponds to the "open" conformation. A cutoff of $12.0 \AA$ was chosen with a default spring constant of 1.0. NumPy ${ }^{44}$ was used to compute the Pearson correlations between the ANM global modes and the experimental distance vectors. We visualized the protein motions with the NMWiz tool in VMD. . $^{39,45}$

Allosteric Pathways. The MCPath Web server was utilized to find allosteric pathways without a set target node. ${ }^{37}$ We generated paths with 10 residue length starting from KRas4B mutational hotspot 12th residue (Gly in wildtype, Asp in G12D and Cys in G12C mutants). Furthermore, we looked for paths between common interface residue Y32 and known allosteric site residue Q99. The Ohm Web server was utilized with the default parameters to perform this allosteric path search. ${ }^{38}$

\section{RESULTS AND DISCUSSION}

KRas4B Interactions Lead to Decreased Flexibility in Global Motions Not Only in the Binding Regions but Also in the Allosteric Lobe. Protein binding is accomplished by mostly new noncovalent bond formation between the interacting residues from two proteins. Moreover, the interactions can affect residues in distal sites by disrupting previous residue communication paths and introducing new ones. This modulation can drastically change the usual residue communication network compared to monomeric states. Such alterations can be revealed by GNM analysis as evidenced with previous NMA studies. ${ }^{31,46,47}$ Here, we applied NMA to reveal the unique dynamics of KRas4B when in complex with an effector or one of the two regulators. We used the monomer KRas4B structures as the reference. The slow modes from 

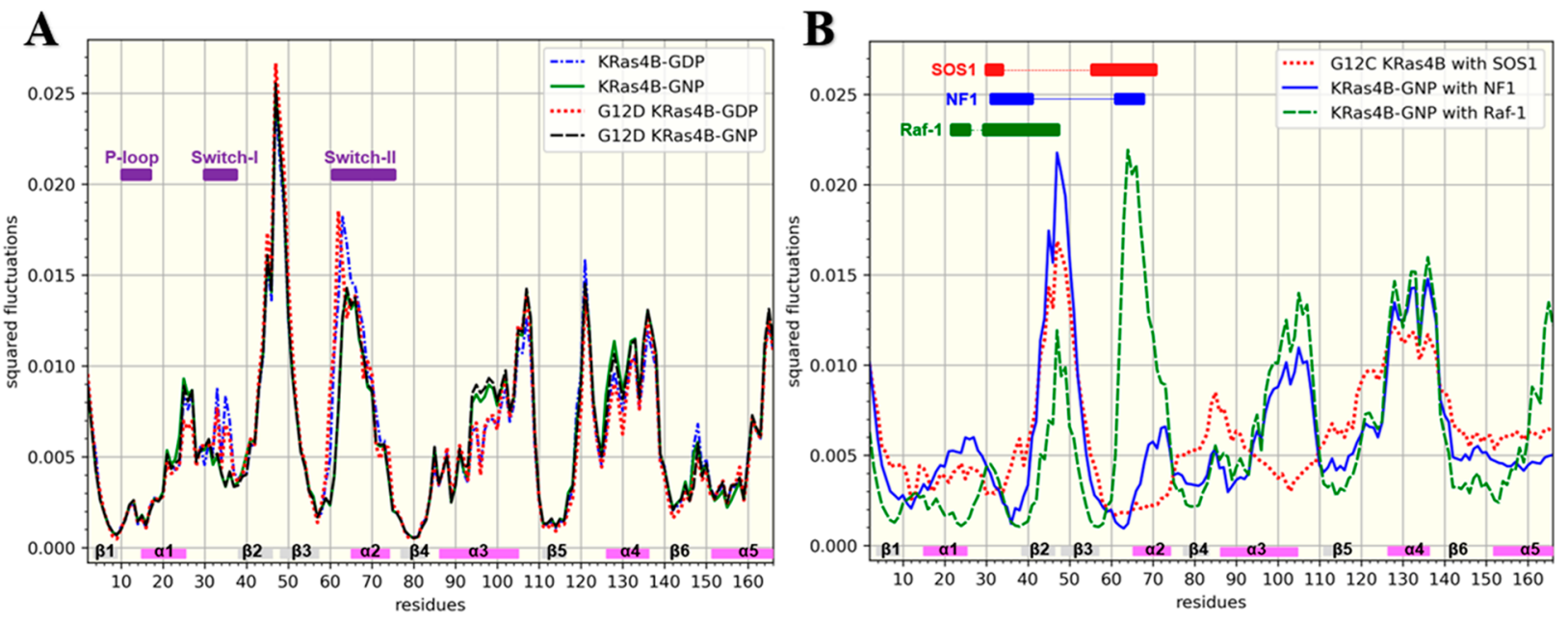

Figure 2. Global protein motions from the slowest 10 modes of GNMs. The weighted squared fluctuations of the unbound KRas4B (A) and protein bound KRas4B (B) are plotted after normalizing the results. The common $\alpha$-helix and $\beta$-sheet residues are indicated above the residue numbers. P-loop, Switch-I, and Switch-II are labeled on the plots along with the main protein interaction interfaces.

GNMs provide the collective residue motions; therefore, the alterations in both interface and distal sites upon binding can be better explained with the top slowest modes. The meansquare fluctuations of the weighted 10 slowest modes are shown in Figure 2 (mode frequencies are provided in Table S2). We identified the hinge sites based on the local minima in the mean-square fluctuation profiles of the proteins which mostly coincide with $\beta 4, \beta 5$, and $\beta 6$ in the monomeric KRas $4 \mathrm{~B}$ (Figure 2A). These core $\beta$-strands contribute to protein stability. Centrality of a residue represents its topological importance in the residue-residue contact network. ${ }^{48}$ Our further topological analysis of KRas4B residues shows that KRas4B residues with highest degree centrality (high number of contacts) overlap with the hinge regions in the monomeric KRas4B (Figure S1A, Table S3).

We find that functionally important residues are affected similarly by partner-binding across all complexes compared to their counterparts in the monomeric state. Protein binding causes the residues in Switch-I, $\beta 2-\mathrm{L} 3-\beta 3$, and L8 (P121) to become less flexible and the residues in $\alpha 4$ to become more flexible in all bound conformations (Figure S2). Switch-I is a common interface for all partner proteins. This might explain the decrease in its flexibility. $\beta 2-\mathrm{L} 3-\beta 3$ is termed the "interswitch region" and has potential roles in both nucleotide-switching and GTP-hydrolysis mechanisms. ${ }^{14,49}$ On the other hand, $\alpha 4$ residues take part in allosteric regulation of Switch-II for GTP hydrolysis with $\alpha 3-\mathrm{L} 7$ residues. ${ }^{13}$ Overall, our approach, using the global motions, can catch functionally important residues of which a subset is already known in the KRas4B dynamics.

We clearly observe that the residue fluctuation changes are at not only the binding interface but also distal sites. Most of the regions with decreased flexibility upon binding correspond to specific interaction interfaces. However, the residues with altered fluctuations in the allosteric lobe are mostly distal to the interacting proteins. Interestingly, the G12C KRas4BSOS1 complex has the most distinct fluctuation profile. SOS1 binding decreases the residue fluctuations in Switch-II and $\alpha 3$ L7 significantly, whereas it increases the residue fluctuations in $\beta 4-\mathrm{L} 6, \beta 5$, and $\beta 6-\mathrm{L} 10-\alpha 5$ (Figure $2 \mathrm{~B}$ ). These results imply that the absence of a nucleotide may potentially lead to a more flexible/open conformation of the protein even compared to the GDP-bound monomer structure. Allosteric shift of $\alpha 3$-L7 enables GTP hydrolysis reaction to take place by Switch-II ordering as explained. ${ }^{13}$ The decreased flexibility in the $\alpha 3$-L7 region is unique to KRas4B-SOS1 interaction. This suggests that the regulatory role of GEFs depends on distinctive dynamics to allow nucleotide exchange.

NF1 inserts an Arg finger to nucleotide binding-site for correct Q61 positioning with P-loop and Switch-I residues around the nucleotide to catalyze the GTP hydrolysis. ${ }^{11,50}$ The obvious reduction in fluctuations of Switch-I and G60-Y64 residues of NF1-bound KRas4B-GNP is most likely a result of NF1 binding. Lu et al. ${ }^{23}$ also found reduced fluctuations when GAP was bound to KRas4B (compared to the unbound KRas4B) using MD simulations. They report Switch and interswitch region fluctuations were suppressed, and we report similar but more pronounced differences for the KRas4BGNP-NF1 complex. Also, KRas4B bound to NF1 has the highest fluctuations in $\alpha 1$ and interswitch region and lowest in G60-Y64 residues compared to Raf-1 or SOS1 bound structures.

Raf- 1 bound KRas4B has the lowest fluctuations in $\alpha 1-\mathrm{L} 2$ and interswitch regions but the highest in Switch-II region. Higher flexibility in Switch-II was also reported for Raf-1 RBD bound HRas complex compared to monomer HRas. ${ }^{51}$ Tran et al. ${ }^{9}$ recently published a KRas4B-Raf-1 structure which included the cysteine-rich domain (CRD). Although the main role of Raf-1 CRD is membrane anchorage, their study showed it is also important for the KRas-Raf- 1 interaction. Raf-1 RBD in this complex interacts with $\alpha 1-\mathrm{L} 2$ residues in KRas4B, whereas its CRD interacts with the interswitch region. We also used this structure in this study. Differential binding of Raf-1 domains to KRas4B can explain the reduced flexibility of the KRas4B interface residues. Baussand and Kleinjung ${ }^{52}$ investigated HRas interactions with different effectors by MD simulations. They found "rigidification" at binding interfaces, which also affected distal sites, and an increase in flexibility at Switch-II, similar to our findings for KRas4B-Raf-1. Moreover, KRas4B in Raf-1 bound conformation has the lowest fluctuations in $\beta 4$ and $\beta 5$ hinge sites but the highest in $\alpha 3$ L7 and $\alpha 4$, even higher than the GAP bound structure (Figure 2B). This is interesting because Raf is also suggested to activate the intrinsic GTP-hydrolysis reaction in Ras proteins. ${ }^{13}$ 

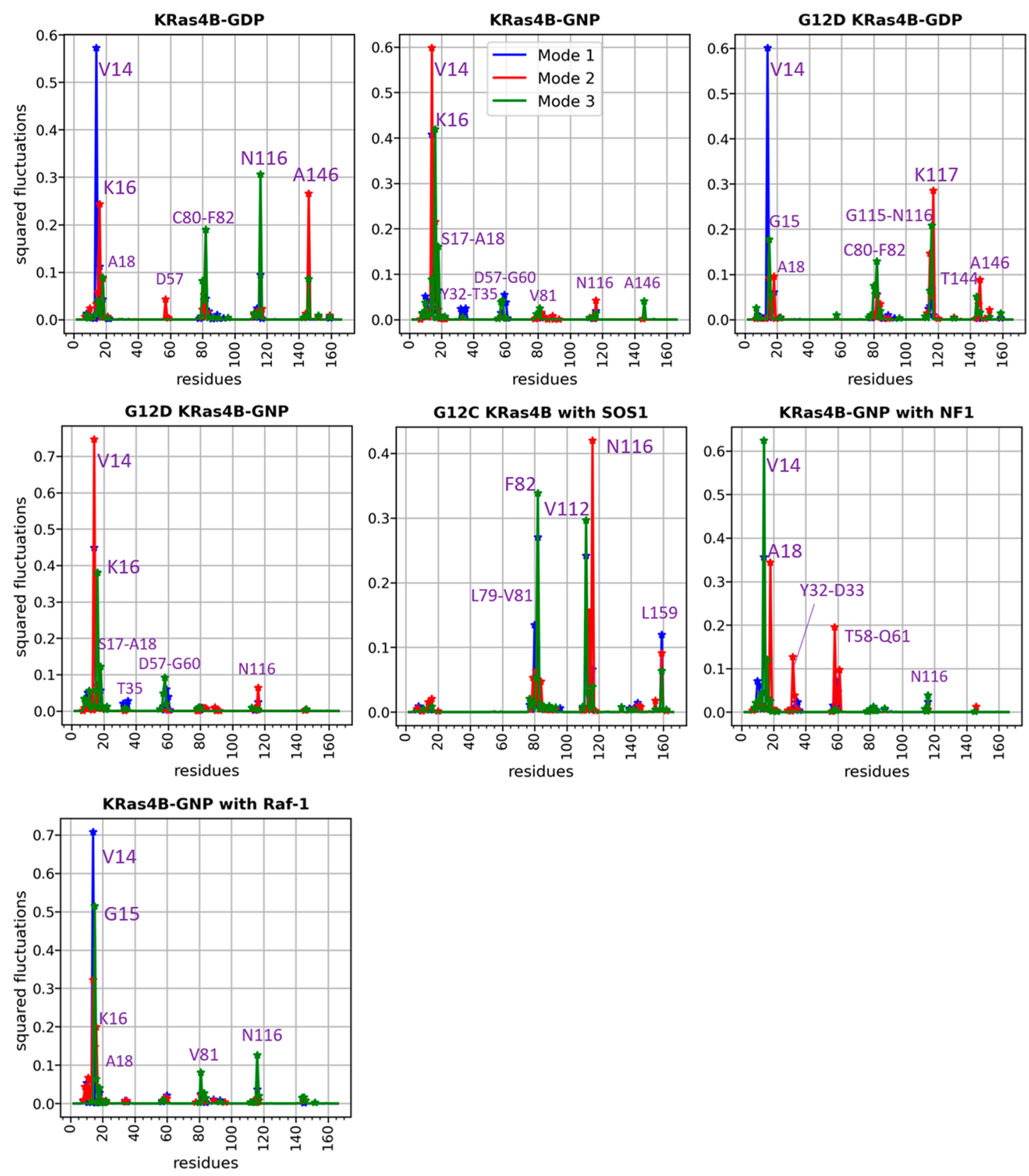

Figure 3. Local protein motions from the three fastest modes of GNMs. The mean-square fluctuations of the individual modes are plotted for each KRas4B structure. The prominent peak site residues are indicated by labels. Refer to Table S5 for the exact residue lists.

Local Motions Reveal Common Hotspot Residues in P-Loop, $\boldsymbol{\beta} 4, \boldsymbol{\beta} 5$-L8, and L10. Besides the global motions, we further investigated the fastest three individual modes of KRas4B in both monomer and complex states (Figure 3). The residues with high fluctuations (peak sites) at the fast modes are potential hotspots. In general, hotspots are critical residues for the stability and functionality of proteins. ${ }^{53}$ We considered a residue as a potential hotspot if it corresponds to a peak site based on the mean-square fluctuation profile calculated from the fastest three modes (Table S5). Among them, some potential hotspots are consistently present in all conformational states. G10-A18 (P-loop), C80-F82 ( $\beta 4), \mathrm{V} 114-\mathrm{K} 117$ $(\beta 5-\mathrm{L} 8)$, and T144-K147 (L10) are regions with potential hotspots in most conformations. $\beta 4$ is at the core of the protein separating the effector and allosteric lobes, and its residues have the highest betweenness (the highest impact in the information flow) in the monomeric structures (Figure S1B, Table S4). Most of the other hotspot residues are involved in nucleotide binding. In addition to P-loop, the nucleotide base binding motifs NKxD (116-119) and ExSAK (143-147) ${ }^{54}$ are populated with hotspots in all conformations. These residues not only are highly fluctuating in the fast modes but also are highly central in the residue-residue contact network. For example, N116 is consistently the most central residue across all conformations. The monomeric KRas4B residues at peak sites also correlate with the residues with high stiffness. ${ }^{14}$ Therefore, we propose these residues as folding hotspots and nucleotide-binding hotspots. Furthermore, the most frequent mutations in KRAS mutant tumors occur at the codons G12, G13, Q61, and A146 (COSMIC data) which are known cancer-driver hotspots. And the hotspots we find either 

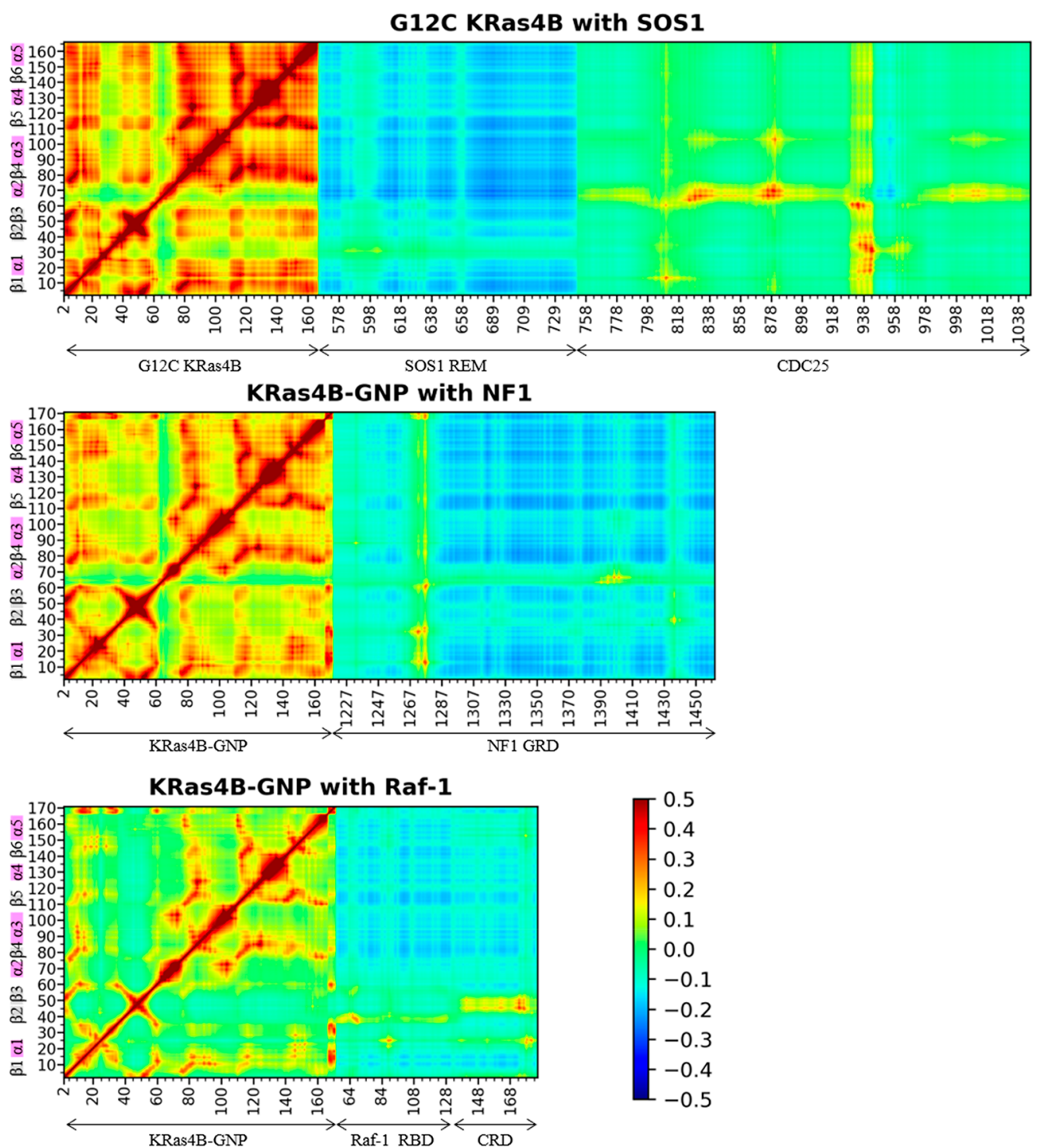

Figure 4. Cross-correlations of protein bound KRas4B. Residue motion correlations were mapped using all GNM normal modes obtained. The interacting protein residues are given on the $x$-axis. Color scale ranges from -0.5 for highly anticorrelated motions (blue) to 0.0 for low or no correlation (green) and to 0.5 for high correlation (red).

coincide with these residues or reside in their proximity in all structures.

The local motions also provide some differences across multiple KRas4B conformations. Peaks at Y32-T35 are only present in KRas4B-GNP monomers and KRas4B-GNP-NF1 complex. This site was proposed as a potential binding pocket previously. ${ }^{55}$ In addition, these Switch-I residues were proposed to be on a Raf RBD and RasGAP binding hotspot cluster. ${ }^{17}$ Although GNM analysis did not reveal hotspots at this site in Raf-1 RBD-CRD bound KRas4B-GNP, it correctly predicted them for NF1 GAP related domain (GRD) bound KRas4B. Local motions further show that G12D KRas4BGDP, G12C KRas4B-SOS1, and KRas4B-GNP-Raf-1 have no peaks at D57-T58 while the others have. GDP-bound monomeric KRas4B and nucleotide-free KRas4B-SOS1 have higher fluctuations in their allosteric lobes compared to the other KRas4B conformations. In fact, the majority of the peaks in SOS1-bound KRas4B are located in the allosteric lobe.

Protein Binding Introduces New Domain Partitioning. We constructed the residue-residue cross-correlation maps shown in Figure 4 using all modes from GNMs. We determine how the correlations between different residue fluctuations are altered in KRas4B to rewire the residue communications upon binding to its partners. The crosscorrelation maps allow a fair comparison between three KRas4B conformations in their complexed states and signify the subdomain regions (like lobes) introduced upon protein binding. Residues at or near the binding interfaces correlate more strongly with the partner protein residues. This leads to a further partitioning of KRas4B lobes at partner-specific locations: both Switch regions in KRas4B-SOS1, Switch-II in KRas4B-GNP-NF1, and the interswitch region in Raf-1 bound Kras4B-GNP. These residues connecting the lobes have also high betweenness centrality which represents a high impact in the information flow in a residue contact network (Figure S1B, Table S4). Additionally, the regulator bound KRas4B complexes have a distinct subdomain formation in the allosteric lobe with a border at D108.

Although the binding of the partners results in increased concerted motions overall (see Figure S3 for cross-correlations 


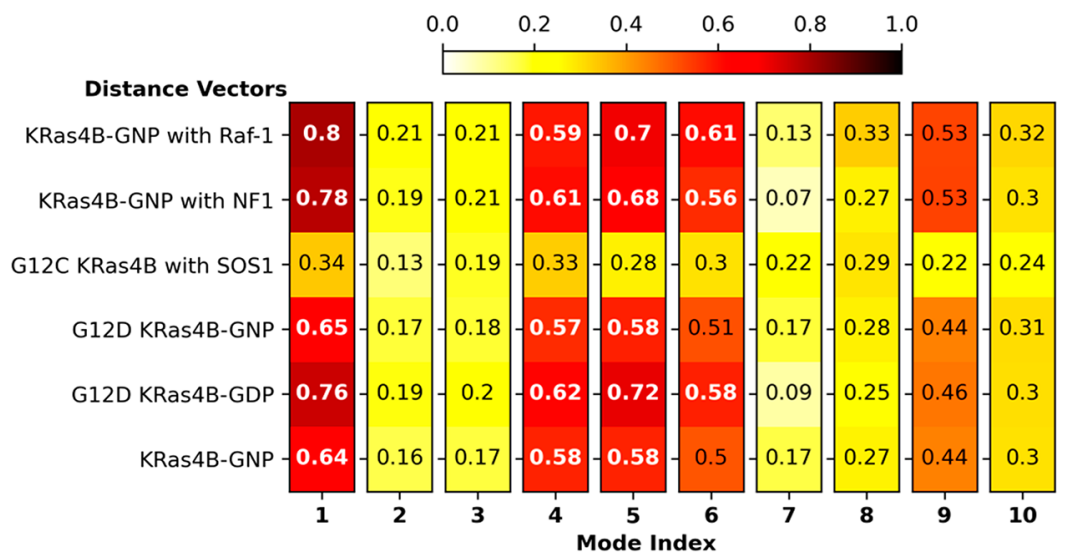

Figure 5. Correlations between the KRas4B-GDP modes from ANM and the experimental distance vectors to the six other structures' main KRas4B chains. The absolute values were used to plot the results, with darker red colors pointing to higher correlation.

of the monomers), the binding interfaces show low intramolecular residue correlations. This might suggest that there is little communication between the Switch regions and the rest of the KRas4B when bound to the regulators. Indeed, the NF1 and SOS1 interactions are to facilitate the hydrolysis and nucleotide exchange reactions. ${ }^{2}$ Also, the KRas4B residues other than in these regions show highly anticorrelated motions with the regulator residues. In the case of KRas4B-GNP-NF1, high intermolecular cross-correlations point to communications involving the nucleotide binding residues G12, Y32, and $\mathrm{G} 60$ in KRas4B. These residues and the bound nucleotide have high positive correlations with the Arg finger residues in NF1 GRD. Additionally, Switch-II residues show correlation with close-by NF1 residues in the complex. These communications may potentially play roles in the GTP hydrolysis reaction as well. SOS1-bound complex in general has KRas4B residues communicating with CDC25 domain (CDC25 homology domain). KRas4B residues in P-loop, Switch-I, Switch-II, and $\alpha 3$ show the highest correlation with $\mathrm{CDC} 25$. These are mostly on the binding interface. However, Switch-I residues in KRas4B also show correlation with SOS1 Ras-exchanger motif (REM) residues, despite not being in proximity to each other. Active state Ras proteins have been noted to bind SOS1 in the REM domain and allosterically regulate the GEF activity of SOS1 in a positive-feedback manner for activation of another Ras. ${ }^{56,57}$ Despite absence of an active Ras protein in the complex we analyzed, it is noteworthy to find such a communication between REM and KRas4B in light of this mechanism.

In contrast to the regulator bound KRas $4 \mathrm{~B}$ complexes, the communication between the allosteric and effector lobes is still strong in Raf-1 bound KRas4B. This might coordinate the membrane interactions required for Ras-effector binding. These are also mediated by KRas4B allosteric lobe residues. ${ }^{58}$ And on that note, the correlation map of Raf- 1 bound KRas4BGNP indicates communications with both RBD and CRD domains of Raf-1. KRas4B Switch-I and interswitch region residues show the most correlated motions with Raf-1, especially with residues in CRD. Raf- 1 RBD has the most correlated motions with KRas4B $\alpha 1$ and $\beta 2$. These also support both previous studies ${ }^{9}$ and our deductions from 10 slowest mode analysis.

KRas4B-GDP Intrinsic Fluctuations Are Correlated with Experimental Conformational Changes. After the detailed analysis of each KRas4B conformational states, we observe how the global motions drive the change to the bound states. HRas deformation modes describing the transition between the "on" and "off" states were previously analyzed in detail, providing information on the nucleotide-switching mechanism in the Ras superfamily. ${ }^{15}$ On the basis of the GNM analysis, we propose that the inactive "open" state KRas4B-GDP modes may describe the transitions to the different protein-bound "closed" conformations. We generated experimental distance vectors from KRas4B-GDP to the other KRas4B structures (see Table S6 for RMSDs) and obtained the residue displacements required for the transitions to their conformations. Since ANM analysis gives the directions of residue motions as well, we used the 10 slowest modes from KRas4B-GDP ANM that could drive these conformational changes (the eigenvalues and mode weightsof the modes analyzed are provided in Table S7). The theoretical residue displacements were compared to the distance vectors. In general, significant correlation was observed as illustrated in Figure 5 (the dark red colors).

Distinct Modes Drive KRas4B to Its Bound Conformational States. Experimental distance vectors are most correlated with mode 1 and then with mode 5 in all except mutant KRas4B-SOS1. This complex has the highest correlation with modes 1 and 4, despite being relatively low. One of the global GNM/ANM modes can successfully drive the open conformations to closed conformations; however, the opposite is not always correct. A combination of several modes, from the most global to intermediate modes, are needed to open an already closed structure owing to the requirement of noncollective localized residue motions. ${ }^{59}$ KRas4B-GDP is an open structure compared to all other structures studied here except KRas4B-SOS1. The mutant KRas4B in SOS1 complex is free of a nucleotide, and its Switch-I is disconnected from the P-loop with the SOS1 interaction that resulted in GDP-exit from the nucleotidebinding site. ${ }^{10}$ Its more "open" conformation would explain why KRas4B-GDP slow modes could not describe the transition to SOS1-bound conformation.

In Figure 6A, the KRas4B-GDP distance vectors to the other six conformations are shown in detail with their most correlated modes' residue fluctuations. The peaks coincide with the most prominent fluctuating regions except for the Switch-I region residues Y32 and T35-I36. Slow modes fail to explain these Switch-I region fluctuations because these residues interact with $\mathrm{Mg}^{2+}$ and $\gamma$-phosphate of GTP in the 
A
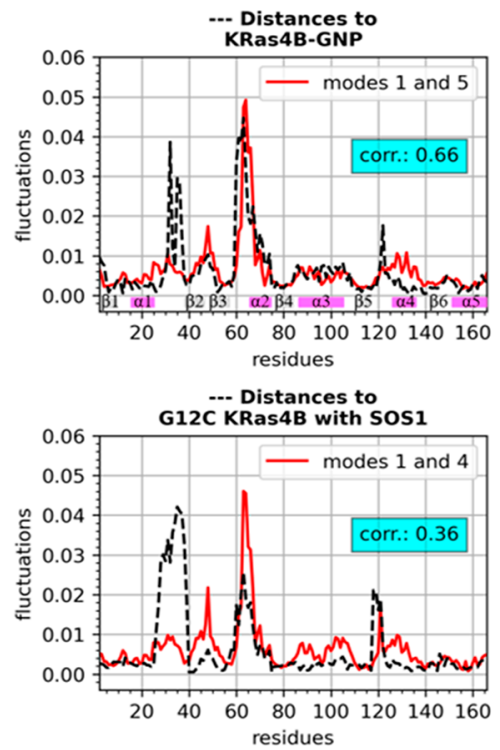

B

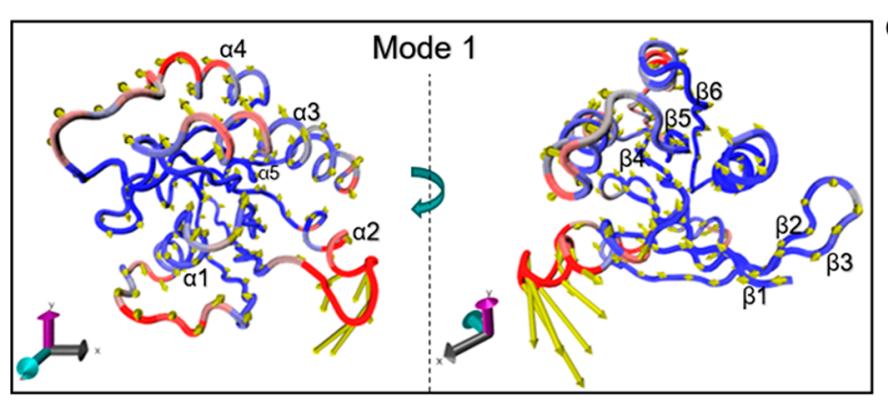

D

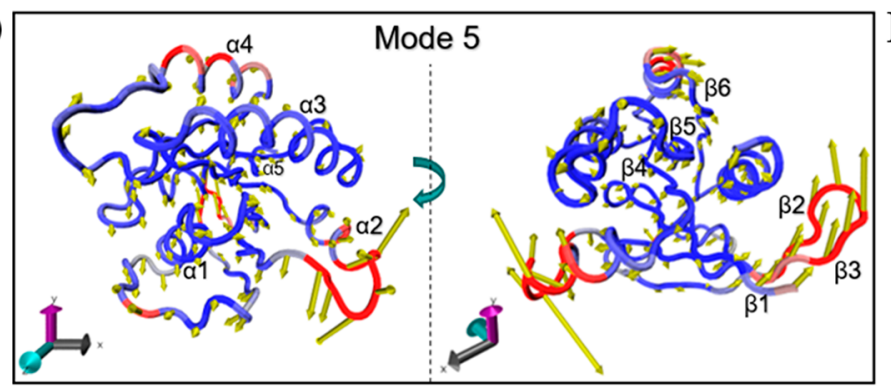

G12D KRas4B-GDP

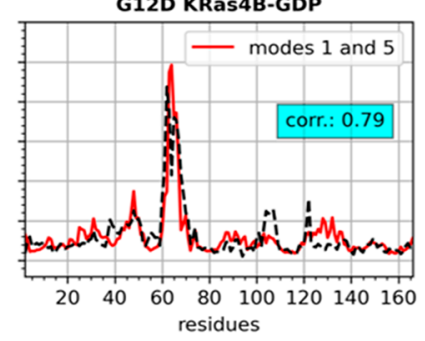

-.- Distances to

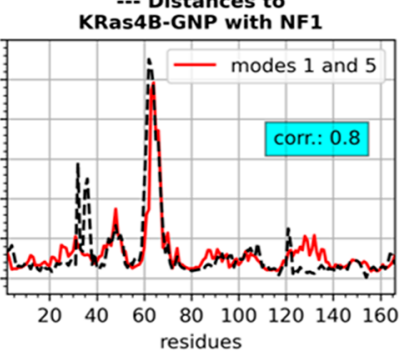

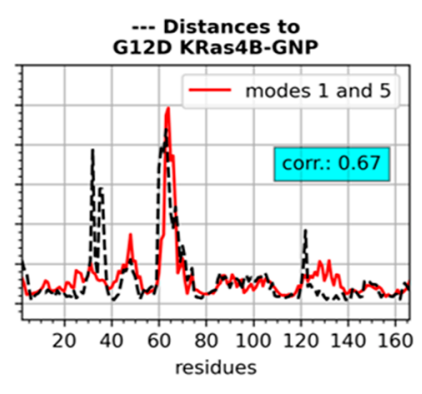

-.- Distances to

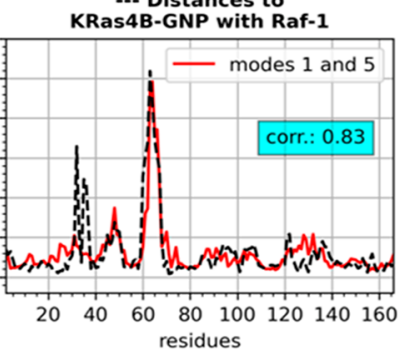

C

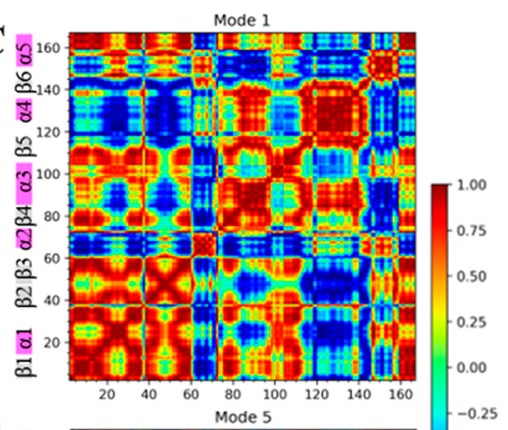

$\mathbf{E}$

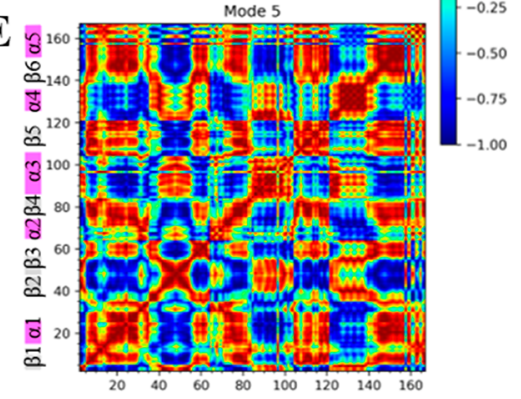

Figure 6. KRas4B-GDP ANM modes most correlated with the experimental distance vectors. (A) KRas4B-GDP distance vectors and their most correlated modes' residue fluctuations are plotted. Mode 1 is drawn on the structure (B), and cross-correlations are mapped (C). Mode 5 is also depicted similarly in (D) and (E). Two perspectives are provided as indicated by the axis orientations (and the rotation). These structure images were rendered with Tachyon ray-tracing in VMD. Blue to gray and red coloring in these represents low to high mobility (color scale was narrowed to highlight the mobile sites), and the arrows indicate the arbitrary motion direction and amplitude. Maps in (C) and (E) have the same color-scale shown on the right; red color means correlated residue motions and blue anticorrelated.

closed structures. ${ }^{12,16,60}$ And probably their fluctuations are more localized and driven by GTP.

KRas4B-GDP Switch-I Shows Anticorrelated Motions with Switch-II and $\alpha \mathbf{4}$ in Mode 1. We mapped the direction of motions derived from eigenvectors of mode 1 onto the KRas4B-GDP structure, together with a color representation based on the amplitude of residue fluctuations (Figure 6B,C). The Switch-II motions are dominant in mode 1, but P-loop, Switch-I, $\alpha 3$ (residues 86-105), part of L8 (residues 120125), $\alpha 4$ (residues 126-136), and L10 (between $\beta 6$ and $\alpha 5$ ) also show some mobility (Figure $6 \mathrm{~B}$ ). We predict that this mode drives a loop closing movement that results in the "closed" conformations of KRas4B. High Switch-II motions correlated with the allosteric lobe, and antiparallel to Switch-I and P-loop motions enable this conformational change.
Meanwhile, $\alpha 4$ and a portion of $\alpha 3$ (until residue 98) move in parallel causing the allosteric lobe to get near the effector lobe. The residue cross-correlations for this mode further show the correlations between these motions in detail (Figure 6C). Switch-I and Switch-II are anticorrelated in this map. Switch-II residues have highly correlated motions with allosteric lobe $\beta 6$ L10- $\alpha 5$ (residues 140-159). While the first part of the effector lobe (residues T2-Q61) displays highly concerted motions as a whole, E37 displays an anticorrelated motion in this part. It rather makes correlated motions with E76-A146 in the allosteric lobe. E37 and D38 normally interact with HVR, ${ }^{7}$ and this could be the reason for the communication between E37 and residues in the allosteric lobe.

Interswitch Region Mobility Is High, and Switch Region Motions Are Correlated in Mode 5. We similarly 


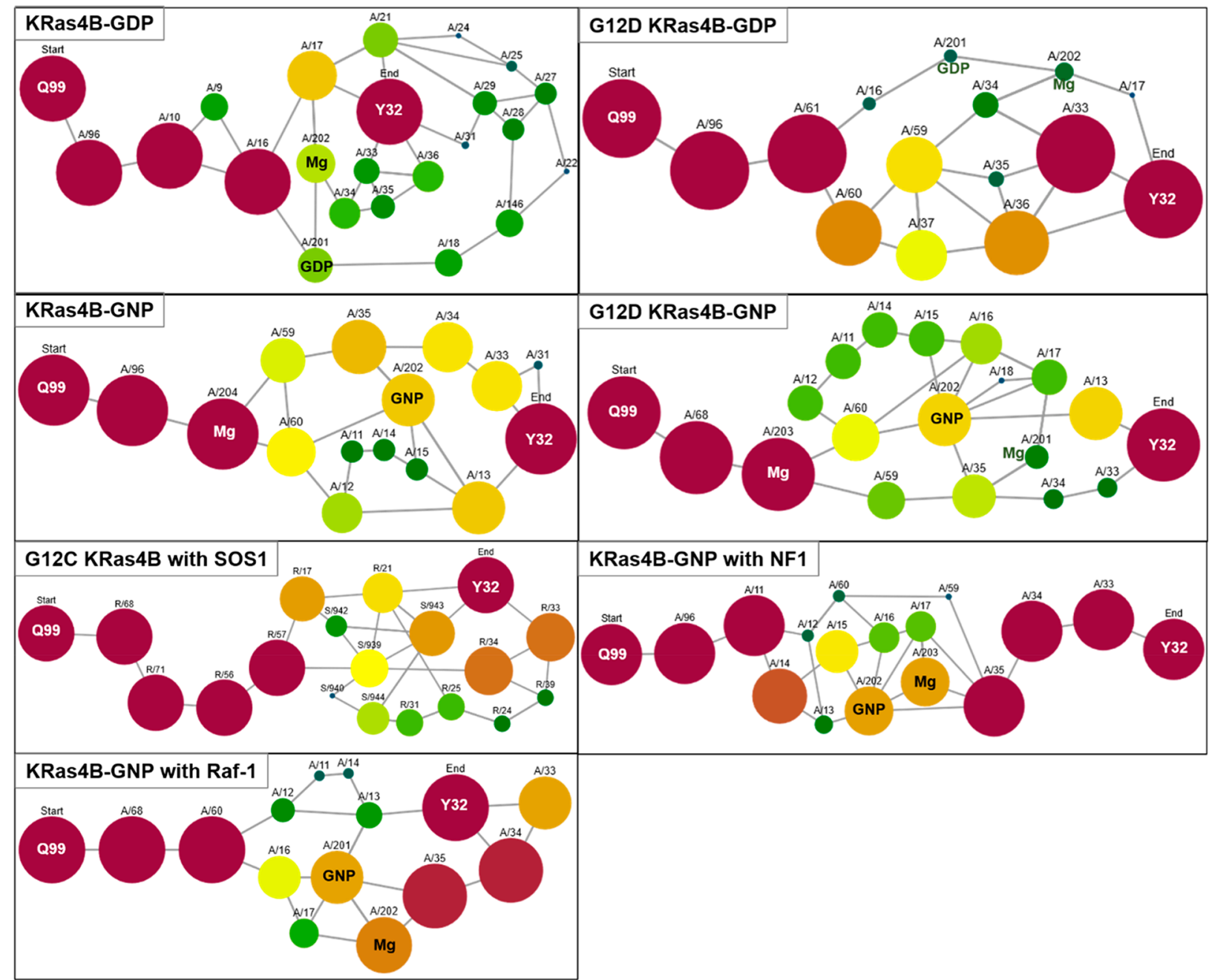

Figure 7. Critical residues in the allosteric pathways found between KRas4B residues Y32 and Q99. The most important 10 pathways were included to obtain the network graphs provided from the $\mathrm{Ohm}$ Web server. The radius and color of each node represent its importance in the signal propagation; red colored big nodes are the most important, while green colored small nodes are the least important nodes. Node labels are in the form of "chain ID/residue index". Chains A and R are KRas4B in the structures analyzed in this study. Start and target nodes, GDP-GNP and Mg ${ }^{2+}$ nodes are additionally labeled on the networks. Refer to Table S9 for the most important paths for each conformation.

show mode 5 in Figure 6D,E. The major difference between mode 5 and mode 1 is the high mobility of the interswitch region $(\beta 2-\mathrm{L} 2-\beta 3$, residues $38-56)$ and lower flexibilities of $\mathrm{P}$ loop and L6- $\alpha 3$. We explained how the interswitch region was affected in both GAP and effector bound KRas4B complexes, and here we see that its motions are also required to describe the conformational changes. Cross-correlations of mode 5 (Figure 6E) reveal that the interswitch region motions are correlated most strongly with each other, A11-V14 (P-loop), T35-D38 (Switch-I), A66 (Switch-II), L6- $\alpha 3$, and P121-P140 (in L8- $\alpha 4-$ L9) residues. Switch-II residues after Y64 show anticorrelated motions with the rest of Switch-II residues. Overall residue fluctuations in the Switch-regions are correlated. They move collectively to reach the closed conformations. Furthermore, the boundary between the allosteric and effector binding lobes is more apparent in this mode.

Allosteric Paths Differ between "Open" and "Closed" Conformations. We further inspected the communication networks in KRas4B structures by analyzing the allosteric paths. The most frequent KRAS oncogenic mutation occurs at the 12th codon. Therefore, we investigated paths starting at this position using MCPath and found residues that bind the nucleotide in the predicted results. The most populated pathways are given in Supporting Information (Table S8). MCPath finds interaction probabilities between any residues with pairwise calculations of their all-atom interaction energies. $^{37}$ Given a perturbation at the 12 th residue of KRas4B, without setting a target node, the signal was propagated to Switch-I in most conformations. The only exceptions are KRas4B-GDP and KRas4B-SOS1, in which the paths remain strictly at the allosteric lobe (L8 and $\beta 4)$. G60 on the other hand, which binds $\gamma$-phosphate of GTP, appears only in the paths of monomeric KRas4B-GNP. KRas4B-GNP-NF1 path goes to an NF1 residue in proximity to Arg finger. Overall, these paths differentiate between the open structures where the P-loop is not strongly coupled to Switch regions but to the $\mathrm{L} 8$ region.

We also investigated paths involving the known KRas4B allosteric site at $\alpha 3$-L7. ${ }^{13}$ We utilized the Ohm server in order to include the bound nucleotide and cofactors. Ohm calculates allosteric paths by using a perturbation propagation algorithm whereby residues are assumed to be in contact with each other based on a chosen cutoff distance. ${ }^{38} \mathrm{Y} 32$ is a common interface 
residue and critical in GTP-hydrolysis as we explained; it forms a hydrogen-bond network around GTP with water molecules, T35 and Q61. There are four proposed allosteric-binding pockets in KRas4B that were validated with experimental research: pocket 1 is a hydrophobic cavity between interswitch and Switch-II; pocket 2 is near Switch-II and $\alpha 3$; pocket 3 is between L7 and $\alpha 5$; and pocket 4 is behind Switch-I. ${ }^{61}$ The Switch-I hotspots we predicted are at pocket 4. Q99 is located in the pocket 2 and propagates signals to Switch-II via a hydrogen-bonding network centered on R68. ${ }^{13}$ Small molecules were found to bind the pocket at this site and inhibit G12C KRas4B. ${ }^{62}$ Therefore, we specifically looked for paths between residues Y32 and Q99. The critical residues found from the most important 10 paths are shown as networks in Figure 7 (most important path nodes are provided in Table S9). These paths include A59, G60, R68, and Y96 residues from the known allosteric pocket and the hydrogen-bond network around it. ${ }^{13,17,62}$ There are also common residues from our MCPath results; connections between P-loop and Switch-I residues of the closed KRas4B conformations are present in $\mathrm{Ohm}$ as well. All structures except KRas4B-SOS1 complex have paths through the nucleotide and $\mathrm{Mg}^{2+}$ nodes as expected, and the importance (weights) of these nodes are higher in the GNP-bound active conformations. This "importance" simply means they appear more often in the paths found (shown with larger nodes colored red in the networks). On the other hand, residues that are seen in only one or few paths are designated with low importance (shown with small nodes colored green). KRas4B-GDP paths alternate between different nodes that are of low importance, including the nucleotide. The signal from the nucleotide is relayed to G60 in the KRas4B-GNP paths. Interestingly, G60 is also a relatively important node in the mutant KRas4B-GDP path; it is connected to Q61, A59, and E37 in this result. Moreover, G60 comes up as a highly important residue only in the Raf- 1 bound KRas4B-GNP pathway and it is of low importance in the Kras4B-GNP-NF1 pathway. In addition, the signal propagated to SOS1 residues in the GEF-bound KRas4B structure. P-loop, Switch-I, and Switch-II are not connected to a nucleotide in this structure, and SOS1 residues appear to make up for the absence of these connections. Therefore, SOS1 might facilitate the signal propagation between active site and allosteric site in this KRas4B conformation. To summarize, these paths connecting the active site residues to the allosteric site $\alpha 3$-L7 show differences in the open and closed conformations.

\section{CONCLUSION}

We explored dynamics of KRas4B in SOS1, NF1, or Raf-1 bound conformational states by normal mode analysis (NMA). KRas4B is known to have two lobes in its G-domain. GNM analysis revealed that these lobes can be further partitioned into subdomains upon binding to its partners. Interface residues with high degree betweenness appear to mediate the sectioning of the effector lobe. These new lobe divisions can be critical in modulating the interaction dynamics with small molecules. Furthermore, KRas4B interactions with different partners suppress the flexibility in not only their binding sites but also distant residues in the allosteric lobe in a partnerspecific way. Interestingly, some of these distal residues have known roles in nucleotide-switching and the intrinsic GTPase activity. In addition, we identified nucleotide binding residues as hotspots in all the structures. ANM analysis of the open state KRas4B-GDP revealed that conformational changes can be driven by the intrinsic residue fluctuations. Moreover, our allosteric path investigation starting at the mutation hotspot G12 showed that this residue was strongly coupled to nucleotide binding residues in either the allosteric lobe or effector lobe in the open and closed states, respectively. The paths reside in Switch-I in the closed stateswhile going to the allosteric lobe in the open states. Paths connecting the common interface residue Y32 to known allosteric site residue Q99 further highlighted differences between the conformations. Overall, NMA helps us in understanding the partnerspecific KRas4B dynamics comprehensively.

\section{ASSOCIATED CONTENT}

\section{Supporting Information}

The Supporting Information is available free of charge at https://pubs.acs.org/doi/10.1021/acs.jpcb.1c00891.

GNM-ANM parameters, GNM mode frequencies, nodecentrality of KRas4B figure, residues with highest degree centrality, residues with highest betweenness centrality, heatmap of mean-square fluctuations from slowest 10 modes, peak site residues in three fastest individual modes, cross-correlations of monomer unbound KRas4B from GNMs, RMSDs of the structures, ANM mode frequencies, MCPath results for paths starting from KRas4B 12th residue, and OHM results for paths between KRas4B Y32 and Q99 (PDF)

\section{AUTHOR INFORMATION}

\section{Corresponding Author}

Ozlem Keskin - Chemical and Biological Engineering, College of Engineering, Koc University, 34450 Istanbul, Turkey; ○ orcid.org/0000-0002-4202-4049; Email: okeskin@ ku.edu.tr

\section{Authors}

Meryem Eren - Molecular Biology and Genetics, Koc University, 34450 Istanbul, Turkey

Nurcan Tuncbag - Chemical and Biological Engineering, College of Engineering, and School of Medicine, Koc University, 34450 Istanbul, Turkey

Hyunbum Jang - Computational Structural Biology Section, Frederick National Laboratory for Cancer Research in the Laboratory of Cancer Immunometabolism, National Cancer Institute, Frederick, Maryland 21702, United States

Ruth Nussinov - Computational Structural Biology Section, Frederick National Laboratory for Cancer Research in the Laboratory of Cancer Immunometabolism, National Cancer Institute, Frederick, Maryland 21702, United States; Department of Human Molecular Genetics and Biochemistry, Sackler School of Medicine, Tel Aviv University, Tel Aviv 69978, Israel

Attila Gursoy - Computer Engineering, Koc University, 34450 Istanbul, Turkey; (1) orcid.org/0000-0002-2297-2113

Complete contact information is available at:

https://pubs.acs.org/10.1021/acs.jpcb.1c00891

\section{Notes}

The authors declare no competing financial interest. 


\section{ACKNOWLEDGMENTS}

O.K. and A.G. are members of Science Academy, Turkey. This project has been funded in whole or in part with federal funds from the National Cancer Institute, National Institutes of Health, under Contract HHSN261200800001E. The content of this publication does not necessarily reflect the views or policies of the Department of Health and Human Services nor does mention of trade names, commercial products, or organizations imply endorsement by the U.S. Government. This research was supported in part by the Intramural Research Program of the NIH, National Cancer Institute, Center for Cancer Research.

\section{REFERENCES}

(1) Wittinghofer, A.; Vetter, I. R. Structure-function relationships of the G domain, a canonical switch motif. Annu. Rev. Biochem. 2011, 80 (1), 943-71.

(2) Bos, J. L.; Rehmann, H.; Wittinghofer, A. GEFs and GAPs: critical elements in the control of small G proteins. Cell 2007, 129 (5), 865-77.

(3) Mo, S. P.; Coulson, J. M.; Prior, I. A. RAS variant signalling. Biochem. Soc. Trans. 2018, 46 (5), 1325-1332.

(4) Hobbs, G. A.; Der, C. J.; Rossman, K. L. RAS isoforms and mutations in cancer at a glance. J. Cell Sci. 2016, 129 (7), 1287-92.

(5) Tate, J. G.; Bamford, S.; Jubb, H. C.; Sondka, Z.; Beare, D. M.; Bindal, N.; Boutselakis, H.; Cole, C. G.; Creatore, C.; Dawson, E.; et al. COSMIC: the Catalogue Of Somatic Mutations In Cancer. Nucleic Acids Res. 2019, 47 (D1), D941-D947.

(6) Pantsar, T. The current understanding of KRAS protein structure and dynamics. Comput. Struct. Biotechnol. J. 2020, 18, 189-198.

(7) Lu, S.; Jang, H.; Muratcioglu, S.; Gursoy, A.; Keskin, O.; Nussinov, R.; Zhang, J. Ras Conformational Ensembles, Allostery, and Signaling. Chem. Rev. 2016, 116 (11), 6607-65.

(8) Ahearn, I. M.; Haigis, K.; Bar-Sagi, D.; Philips, M. R. Regulating the regulator: post-translational modification of RAS. Nat. Rev. Mol. Cell Biol. 2012, 13 (1), 39-51.

(9) Tran, T. H.; Chan, A. H.; Young, L. C.; Bindu, L.; Neale, C.; Messing, S.; Dharmaiah, S.; Taylor, T.; Denson, J.-P.; Esposito, D.; et al. KRAS interaction with RAF1 RAS-binding domain and cysteinerich domain provides insights into RAS-mediated RAF activation. Nat. Commun. 2021, 12 (1), 1176.

(10) Boriack-Sjodin, P. A.; Margarit, S. M.; Bar-Sagi, D.; Kuriyan, J. The structural basis of the activation of Ras by Sos. Nature 1998, 394 (6691), 337-43.

(11) Rabara, D.; Tran, T. H.; Dharmaiah, S.; Stephens, R. M.; McCormick, F.; Simanshu, D. K.; Holderfield, M. KRAS G13D sensitivity to neurofibromin-mediated GTP hydrolysis. Proc. Natl. Acad. Sci. U. S. A. 2019, 116 (44), 22122-22131.

(12) Li, Y.; Zhang, Y.; Grosseruschkamp, F.; Stephan, S.; Cui, Q.; Kotting, C.; Xia, F.; Gerwert, K. Specific Substates of Ras To Interact with GAPs and Effectors: Revealed by Theoretical Simulations and FTIR Experiments. J. Phys. Chem. Lett. 2018, 9 (6), 1312-1317.

(13) Buhrman, G.; Holzapfel, G.; Fetics, S.; Mattos, C. Allosteric modulation of Ras positions Q61 for a direct role in catalysis. Proc. Natl. Acad. Sci. U. S. A. 2010, 107 (11), 4931-6.

(14) Vatansever, S.; Gumus, Z. H.; Erman, B. Intrinsic K-Ras dynamics: A novel molecular dynamics data analysis method shows causality between residue pair motions. Sci. Rep. 2016, 6, 37012.

(15) Raimondi, F.; Orozco, M.; Fanelli, F. Deciphering the deformation modes associated with function retention and specialization in members of the Ras superfamily. Structure 2010, 18 (3), 402-14.

(16) Matsumoto, S.; Miyano, N.; Baba, S.; Liao, J.; Kawamura, T.; Tsuda, C.; Takeda, A.; Yamamoto, M.; Kumasaka, T.; Kataoka, T.; Shima, F. Molecular Mechanism for Conformational Dynamics of
Ras.GTP Elucidated from In-Situ Structural Transition in Crystal. Sci. Rep. 2016, 6, 25931.

(17) Buhrman, G.; O'Connor, C.; Zerbe, B.; Kearney, B. M.; Napoleon, R.; Kovrigina, E. A.; Vajda, S.; Kozakov, D.; Kovrigin, E. L.; Mattos, C. Analysis of binding site hot spots on the surface of Ras GTPase. J. Mol. Biol. 2011, 413 (4), 773-89.

(18) Shima, F.; Ijiri, Y.; Muraoka, S.; Liao, J.; Ye, M.; Araki, M.; Matsumoto, K.; Yamamoto, N.; Sugimoto, T.; Yoshikawa, Y.; et al. Structural basis for conformational dynamics of GTP-bound Ras protein. J. Biol. Chem. 2010, 285 (29), 22696-705.

(19) Vatansever, S.; Erman, B.; Gumus, Z. H. Comparative effects of oncogenic mutations G12C, G12V, G13D, and $\mathrm{Q} 61 \mathrm{H}$ on local conformations and dynamics of K-Ras. Comput. Struct. Biotechnol. J. 2020, 18, 1000-1011.

(20) Poulin, E. J.; Bera, A. K.; Lu, J.; Lin, Y. J.; Strasser, S. D.; Paulo, J. A.; Huang, T. Q.; Morales, C.; Yan, W.; Cook, J.; et al. TissueSpecific Oncogenic Activity of KRAS(A146T). Cancer Discovery 2019, 9 (6), $738-755$.

(21) Pantsar, T.; Rissanen, S.; Dauch, D.; Laitinen, T.; Vattulainen, I.; Poso, A. Assessment of mutation probabilities of KRAS G12 missense mutants and their long-timescale dynamics by atomistic molecular simulations and Markov state modeling. PLoS Comput. Biol. 2018, 14 (9), e1006458.

(22) Sayyed-Ahmad, A.; Prakash, P.; Gorfe, A. A. Distinct dynamics and interaction patterns in $\mathrm{H}$ - and K-Ras oncogenic P-loop mutants. Proteins: Struct., Funct., Genet. 2017, 85 (9), 1618-1632.

(23) Lu, S.; Jang, H.; Nussinov, R.; Zhang, J. The Structural Basis of Oncogenic Mutations G12, G13 and Q61 in Small GTPase K-Ras4B. Sci. Rep. 2016, 6, 21949.

(24) Bahar, I.; Rader, A. J. Coarse-grained normal mode analysis in structural biology. Curr. Opin. Struct. Biol. 2005, 15 (5), 586-92.

(25) Bahar, I.; Atilgan, A. R.; Erman, B. Direct evaluation of thermal fluctuations in proteins using a single-parameter harmonic potential. Folding Des. 1997, 2 (3), 173-181.

(26) Atilgan, A. R.; Durell, S. R.; Jernigan, R. L.; Demirel, M. C.; Keskin, O.; Bahar, I. Anisotropy of fluctuation dynamics of proteins with an elastic network model. Biophys. J. 2001, 80 (1), 505-15.

(27) Bahar, I.; Atilgan, A. R.; Demirel, M. C.; Erman, B. Vibrational dynamics of folded proteins: Significance of slow and fast motions in relation to function and stability. Phys. Rev. Lett. 1998, 80 (12), 2733-2736.

(28) Yang, L. W.; Bahar, I. Coupling between catalytic site and collective dynamics: A requirement for mechanochemical activity of enzymes. Structure 2005, 13 (6), 893-904.

(29) Ozbek, P.; Soner, S.; Haliloglu, T. Hot Spots in a Network of Functional Sites. PLoS One 2013, 8 (9), e74320.

(30) Demirel, M. C.; Atilgan, A. R.; Jernigan, R. L.; Erman, B.; Bahar, I. Identification of kinetically hot residues in proteins. Protein Sci. 1998, 7 (12), 2522-2532.

(31) Tandon, H.; de Brevern, A. G.; Srinivasan, N. Transient association between proteins elicits alteration of dynamics at sites far away from interfaces. Structure 2021, 29, 371-384.

(32) Zhang, Y.; Doruker, P.; Kaynak, B.; Zhang, S.; Krieger, J.; Li, H.; Bahar, I. Intrinsic dynamics is evolutionarily optimized to enable allosteric behavior. Curr. Opin. Struct. Biol. 2020, 62, 14-21.

(33) Atilgan, A. R.; Atilgan, C. Local motifs in proteins combine to generate global functional moves. Briefings Funct. Genomics 2012, 11 (6), 479-88.

(34) Kaynak, B. T.; Findik, D.; Doruker, P. RESPEC Incorporates Residue Specificity and the Ligand Effect into the Elastic Network Model. J. Phys. Chem. B 2018, 122 (21), 5347-5355.

(35) Ross, C. J.; Atilgan, A. R.; Tastan Bishop, Ö.; Atilgan, C. Unraveling the Motions behind Enterovirus 71 Uncoating. Biophys. J. 2018, 114 (4), 822-838.

(36) Gerek, Z. N.; Ozkan, S. B. Change in allosteric network affects binding affinities of PDZ domains: analysis through perturbation response scanning. PLoS Comput. Biol. 2011, 7 (10), e1002154.

(37) Kaya, C.; Armutlulu, A.; Ekesan, S.; Haliloglu, T. MCPath: Monte Carlo path generation approach to predict likely allosteric 
pathways and functional residues. Nucleic Acids Res. 2013, 41 (W1), W249-W255.

(38) Wang, J.; Jain, A.; McDonald, L. R.; Gambogi, C.; Lee, A. L.; Dokholyan, N. V. Mapping allosteric communications within individual proteins. Nat. Commun. 2020, 11 (1), 3862.

(39) Bakan, A.; Meireles, L. M.; Bahar, I. ProDy: protein dynamics inferred from theory and experiments. Bioinformatics 2011, 27 (11), $1575-7$.

(40) Hunter, J. D. Matplotlib: A 2D graphics environment. Comput. Sci. Eng. 2007, 9 (03), 90-95.

(41) Cukuroglu, E.; Gursoy, A.; Keskin, O. HotRegion: a database of predicted hot spot clusters. Nucleic Acids Res. 2012, 40 (D1), D829D833.

(42) Chakrabarty, B.; Naganathan, V.; Garg, K.; Agarwal, Y.; Parekh, N. NAPS update: network analysis of molecular dynamics data and protein-nucleic acid complexes. Nucleic Acids Res. 2019, 47 (W1), W462-W470.

(43) Ozgur, B.; Ozdemir, E. S.; Gursoy, A.; Keskin, O. Relation between Protein Intrinsic Normal Mode Weights and Pre-Existing Conformer Populations. J. Phys. Chem. B 2017, 121 (15), 3686-3700.

(44) Harris, C. R.; Millman, K. J.; van der Walt, S. J.; Gommers, R.; Virtanen, P.; Cournapeau, D.; Wieser, E.; Taylor, J.; Berg, S.; Smith, N. J.; et al. Array programming with NumPy. Nature 2020, 585 (7825), 357-362.

(45) Humphrey, W.; Dalke, A.; Schulten, K. VMD: visual molecular dynamics. J. Mol. Graphics 1996, 14 (1), 33-8.

(46) Sorkina, T.; Cheng, M. H.; Bagalkot, T. R.; Wallace, C.; Watkins, S. C.; Bahar, I.; Sorkin, A. Direct coupling of oligomerization and oligomerization-driven endocytosis of the dopamine transporter to its conformational mechanics and activity. J. Biol. Chem. 2021, 296, 100430.

(47) Keskin, O. Binding induced conformational changes of proteins correlate with their intrinsic fluctuations: a case study of antibodies. BMC Struct. Biol. 2007, 7, 31.

(48) Chakrabarty, B.; Parekh, N. NAPS: Network Analysis of Protein Structures. Nucleic Acids Res. 2016, 44 (W1), W375-W382.

(49) Raimondi, F.; Portella, G.; Orozco, M.; Fanelli, F. Nucleotide binding switches the information flow in ras GTPases. PLoS Comput. Biol. 2011, 7 (3), e1001098.

(50) Kotting, C.; Kallenbach, A.; Suveyzdis, Y.; Wittinghofer, A.; Gerwert, K. The GAP arginine finger movement into the catalytic site of Ras increases the activation entropy. Proc. Natl. Acad. Sci. U. S. A. 2008, 105 (17), 6260-5.

(51) Fetics, S. K.; Guterres, H.; Kearney, B. M.; Buhrman, G.; Ma, B.; Nussinov, R.; Mattos, C. Allosteric effects of the oncogenic RasQ61L mutant on Raf-RBD. Structure 2015, 23 (3), 505-516.

(52) Baussand, J.; Kleinjung, J. Specific Conformational States of Ras GTPase upon Effector Binding. J. Chem. Theory Comput. 2013, 9 (1), $738-749$.

(53) Haliloglu, T.; Keskin, O.; Ma, B.; Nussinov, R. How similar are protein folding and protein binding nuclei? Examination of vibrational motions of energy hot spots and conserved residues. Biophys. J. 2005, 88 (3), 1552-1559.

(54) Valencia, A.; Chardin, P.; Wittinghofer, A.; Sander, C. The ras protein family: evolutionary tree and role of conserved amino acids. Biochemistry 1991, 30 (19), 4637-48.

(55) Grant, B. J.; Lukman, S.; Hocker, H. J.; Sayyah, J.; Brown, J. H.; McCammon, J. A.; Gorfe, A. A. Novel Allosteric Sites on Ras for Lead Generation. PLoS One 2011, 6 (10), e25711.

(56) Sondermann, H.; Soisson, S. M.; Boykevisch, S.; Yang, S. S.; Bar-Sagi, D.; Kuriyan, J. Structural analysis of autoinhibition in the Ras activator Son of sevenless. Cell 2004, 119 (3), 393-405.

(57) Margarit, S. M.; Sondermann, H.; Hall, B. E.; Nagar, B.; Hoelz, A.; Pirruccello, M.; Bar-Sagi, D.; Kuriyan, J. Structural evidence for feedback activation by Ras.GTP of the Ras-specific nucleotide exchange factor SOS. Cell 2003, 112 (5), 685-95.

(58) Jang, H.; Zhang, M.; Nussinov, R. The quaternary assembly of KRas4B with Raf-1 at the membrane. Comput. Struct. Biotechnol. J. 2020, 18, 737-748.
(59) Tama, F.; Sanejouand, Y. H. Conformational change of proteins arising from normal mode calculations. Protein Eng., Des. Sel. 2001, 14 (1), $1-6$.

(60) Rudack, T.; Xia, F.; Schlitter, J.; Kotting, C.; Gerwert, K. The role of magnesium for geometry and charge in GTP hydrolysis, revealed by quantum mechanics/molecular mechanics simulations. Biophys. J. 2012, 103 (2), 293-302.

(61) Prakash, P.; Hancock, J. F.; Gorfe, A. A. Binding hotspots on Kras: consensus ligand binding sites and other reactive regions from probe-based molecular dynamics analysis. Proteins: Struct., Funct., Genet. 2015, 83 (5), 898-909.

(62) Ostrem, J. M.; Peters, U.; Sos, M. L.; Wells, J. A.; Shokat, K. M. K-Ras(G12C) inhibitors allosterically control GTP affinity and effector interactions. Nature 2013, 503 (7477), 548-51. 